\title{
Exploring the Stresses and Health of the Nurses in the Psychiatric Hospitals in Eastern Taiwan: Does the Leisure Destination Location Help Nurses Cope with
} Stress?

\section{Feng-Chuan Pan $^{1 *}$ and Sen-Jih Chen ${ }^{2}$}

${ }^{1}$ Tajen University, Pingtung, Taiwan

${ }^{2}$ Bachelor, Pao-Chien Hospital, Taiwan

\begin{abstract}
Background: It has been suggested that nurses who care for the mentally ill patients are exposed to increased stress and have poorer health status. Leisure and tourism have been proposed to be effective sources of stress coping. The purpose of this research was to investigate stress and health of nurses working in the psychiatric hospitals in the eastern Taiwan, which was known for its excellent tourism resources.

Methods: Samples were purposively selected from the nursing staffs of two major psychiatry hospitals in the region. Overall, 333 valid responses were gathered using Short Form-36 (SF-36) for health status and the Nursing Staffs Pressure Scale measuring job stressors.

Results: The perceived job stress varied significantly by age and job position. The perceived health status varied significantly by services, seniorities, marital, and numbers of children. The job stress correlated negatively with the health status. Family obligation and person-environment fit may explain why nurses in this particular area experience higher stress and poorer health status compared to their colleagues in the west.

Conclusion: Although nurses in the eastern Taiwan receive relatively attractive compensation package and enjoy social status in an economically less developed area with abundant tourism resources, they experience higher stress and worse health. Nursing professionals with higher socio-economic status are in fact inversely exposed to greater stress, of which detrimental to their levels of person-environment fit. Since the leisure and tourism resources may not be effective alternatives for coping with stress and the family obligation has a strong association with the poor health status, a supportive system is apparently needed.
\end{abstract}

Keywords: Psychiatric nurse; Job stress; Quality of life; Family obligation; Person-environment fit

\section{Introduction}

Nurses are inevitably exposed to an extremely stressful work environment [1-4], which in turn causes health risks, turnover, and accordingly the productivity loss to the hospitals [1]. Taiwan launched a new hospital accreditation system in 2007, which requires higher levels of caring procedures and excessive administrative works. Recent studies have shown that stress causes many health problems, such as headache, back pain, joint pain, anxiety, hypertension, and some of other somatic symptoms or cardiovascular problems [3-7]. These influence the nurses' morale and hospitals' service quality and productivity [4-11].

The perceptions of stress may vary across different demographic characteristics, including job position, seniority, experience, nature of the work (unit, shift, etc.), age, education, marital status [2-5]. Taking vacation and engaging in leisure activities be perceived as the stress coping strategies that ease the negative effects of stress $[9,10,12]$. Would nurses who work in a hospital located in a tourism destination that offers plenty of leisure resources experience less stress and better health? To our best knowledge, few studies, if any, have specifically addressed stress among psychiatric nurses in this kind of context $[4,11,12]$.

Scientific studies on stress attracted scholars from a wide variety of disciplines; yet, mainstream studies focus on the interaction of personal reactions with the job environment $[2,8,9,11]$, suggesting that the "fit" between someone's personal qualities and his/her job environment may determine the magnitude of job stress. The higher the fit between the person and his/her work environment (PE fit), the lower the possibility and magnitude of job stress $[13,14]$. We may infer that if the individual works in a place where the members are highly similar to each other in terms of professional knowledge, training, and capability and, in many cases, offer supplementary hospital services [12], then the congruence between individual professional capabilities and job environments tends to be high [15]. Ironically, job stress, as perceived by the nurses in hospitals was high, and turnover rate increased accordingly [11].

As Schneider noted, the PE fit should include at least the national culture in the relation between the individual's PE fit and affective outcome $[15,16]$. Previous studies have generally agreed that western culture is more individually oriented, wherein an individual is separate from the environment, whereas the Confucian Oriental model is relational and "selfhood- oriented", wherein an individual is relational, behaves in response to others, and is self-cultivating in response to the social environment $[13,15]$. Since an individual's job stress is aligned with her or his subjective perception of PE fit, and to take the regional subculture may exist in the Easter Taiwan, we further explore how the $\mathrm{P}-\mathrm{E}$ fit in this particular region affect the perceived stress and health.

Two hypotheses were then developed based on the literature review, as discussed in the introduction section.

*Corresponding author: Feng-Chuan Pan, Tajen University, Pingtung, Taiwan, Tel: 886-932-808695; E-mail:profpan900@gmail.com

Received February 05, 2015; Accepted March 30, 2015; Published April 07, 2015

Citation: Pan FC, Chen SJ (2015) Exploring the Stresses and Health of the Nurses in the Psychiatric Hospitals in Eastern Taiwan: Does the Leisure Destination Location Help Nurses Cope with Stress? J Psychiatry 18: 277 doi:10.4172/ psychiatry. 1000277

Copyright: ( 2015 Pan FC, et al. This is an open-access article distributed under the terms of the Creative Commons Attribution License, which permits unrestricted use, distribution, and reproduction in any medium, provided the original author and source are credited 
H1: Perceived stress levels and health status will differ significantly by respondents' demographic and job characteristics.

H2: The perceived health status is negatively correlated with the perceived stress.

\section{Materials and Methods}

\section{Materials}

The sample includes nurses from two major psychiatric teaching hospitals in the city of Yuli in Eastern Taiwan with scattered population and abundant natural sightseeing resources. Samples included nurses working in outpatient, inpatient, and acute departments, regardless of gender or ethnicity. Nurses included are registered and enrolled nurses, nursing officers, nurse practitioners. Nurses working on a part-time basis were excluded. One hospital (HS) is state owned, and it admits psychiatric patients from the entire state. The other hospital (HV) was established by the Veteran Affairs Commission for veterans, and it is open to veterans as well as the public with psychiatric problems. With the permission of the hospital directors, all questionnaires were distributed and collected through nurse supervisors of respective hospitals. To comply with the code of ethics of research, an informed consent form was printed on the first page of the questionnaire. It contained the information about the research and advised the respondents that they may reject or withdraw from the study at any time with their own will. In HS, two hundred (200) questionnaires out of which 155 contained valid responses for a response rate of $77.5 \%$. In HV, two hundred and fifty (250) were distributed and 176 contained valid responses for a response rate of $71.2 \%$. The grand total response rate was $74.0 \%(333 / 450)$. A chi-square goodness-of-test analysis result showed that the observations did not differ by gender $\left(\mathrm{X}^{2}=0.678<3.84\right.$, $\mathrm{df}=1, \alpha=0.05$ ) in the sample of enrolled nurses (male $1.80 \%$ and female 98.20\%, Taiwan Union of Nurse Association, 2014) [17].

\section{Methods}

This cross-sectional research adopted structured questionnaire to collect the data for analysis. A questionnaire containing two scales was used to collect the relevant data as well as demographic and job characteristics. Chinese version of the Nurse Stress Checklist was used to measure stress levels. It has been found to have acceptable reliability and validity in Taiwan $[18,19]$. The current research adopted the Chinese version of Short Form-36 (SF-36) to test the respondents' perceived health status. It has been found to be reliable and valid $[20,21]$. The data gathered were analyzed using statistical techniques of descriptive analysis, t-test, one-way ANOVA, and Pearson's Correlation using SPSS for Windows 17.0.

\section{Results}

\section{Sample description}

Of the 333 respondents, 4 (1.2\%) were male, as shown in Table 1. Most respondents were 26-30 years of age. Furthermore, $23.78 \%$ worked at the state-owned and $23.03 \%$ at the veteran hospital. Considering both hospitals, around $93 \%$ of the respondents of the current research were 40 years old or younger. Nearly $100 \%$ of the respondents were well educated with at least an associate degree, and over $60 \%$ of the respondents had a bachelor or higher degree. More than half of the nurses in these hospitals were single, 91 (58.71\%) in HS and 93 (53.37\%) in HV.

Most respondents earned 600 thousands NTD or lower (around 20,000 USD) per year. Among them, senior nurses were generally paid more than $700 \mathrm{~K}$ per annum. This salary is considered high in the Eastern Taiwan and is sufficient to lead a good life in Eastern Taiwan, where the average income can be as low as $300 \mathrm{~K}$ in a less developed region. HS staffs have a higher proportion of junior employees, even though it was established more than 40 years ago while $\mathrm{HV}$ was established less than 20 years ago. There is no evidence to suggest that nurses in HS are exposed to higher stress compared to HV.

\section{Description of stress and health}

Although nurses in HV experienced higher stress compared to those in HS, these levels of stress were not severe, as shown in Table 2. The average stress score for the entire sample of respondents was 4.01 (s.d.=2.24) on a 9-point (0-8) scale, with average score of $3.83(2.19)$ for HV and average score of 4.16 (2.21) for HS. "Personal response" seems to be the major contribution to stress among the nurses of both hospitals, with average scores of 4.29(1.96) for HS and 4.43 (1.61) for HV. "Feel tired" is the most notable item with the mode at "7" for HS and " 8 " for HV. The least stress nurses perceived in this research is in the dimension of competence, with a grand total average of 3.27 (1.34). This may imply that the respondents were well trained and skillful. Interestingly, nurses in HS obtained the lowest score of 3.36 (1.72) in the dimension of work concerns. This dimension comprises factors in the patient caring area, such as interactions (with physicians, patients, and other colleagues), job content, and time allocation. It is interesting to note that time pressure is the major contributor to stress among both hospitals' nurses. "Physical function" is the highest scoring dimension of the eight dimensions for nurses at case hospitals, with HS obtaining 80.87 points on a 100 points scale and HV obtaining 81.32 and with the grand average of 81.11. A higher score denotes a superior outcome from health status self-assessment. The second dimension is the issue of bodily pain. The moderate scores on this item imply that nurses do not experience body pain problems. Ironically, nurses assessed their health status as low in terms of vitality (45.45), general health (49.04), and mental health (53.91). It has to be noted that these nurses are well trained and experienced in taking care of mentally ill patients. A worried expert might find it difficult to provide satisfactory quality of care.

\section{Demographic factors in stress and health status}

Subsequently, we examined differences in stress and health status in terms of demographic factors, such as age, education, tenure, marital status, number of children, annual income, and job factors, such as hospitals, units, and work shift as independent variables. We performed independent $t$-test and one-way ANOVA to test the differences among independent variables in perceived health and stress levels, respectively. As Table 3 shows, the hospital did not differentiate stress and health status, as t-score was not statistically significant at $\mathrm{p}<0.05$.

The results further showed that stress levels varied as a function of the respondents' seniority, unit, work shift, marital status, and number of children. Perceived health status differs according to the person's education and seniority. Hypothesis one was therefore supported.

\section{Link stress and health status}

The possible relationships between stress and health status form the core of the research. Perceived health and associated dimensions were the dependent variables, and the job stress and associated dimensions were the independent variables. The test results of Pearson's correlations are shown in Table 4 . As we predicted, job stress correlated negatively with health status $(r=-0.406)$. The fact that excess stress is harmful to people's health also applies to the nurses in a psychiatric hospital. Most health dimensions correlated also highly with the self-assessed 
Citation: Pan FC, Chen SJ (2015) Exploring the Stresses and Health of the Nurses in the Psychiatric Hospitals in Eastern Taiwan: Does the Leisure Destination Location Help Nurses Cope with Stress?. J Psychiatry 18: 277 doi:10.4172/psychiatry. 1000277

Page 3 of 6

health status, except for mental health. Interestingly, mental health had the weakest and positive correlation with health status, which seems strange in a psychiatric hospital whose employees are supposed to be mentally strong. The personal responses to factor stress had high negative correlation with several dimensions of health status, for example, perceived health $(\mathrm{r}=-0.696)$, vitality $(\mathrm{r}=-0.679)$, and emotional limitation $(\mathrm{r}=-0.511)$. This dimension represents global physical and psychological manifestations and has much stronger relationships with all aspects of health status compared to other dimensions. As shown in Table 4, hypothesis two of the current research was thus supported. It is interesting to note that professional competence (PC) did not correlate significantly with "unable to complete assignment (PP)," "personal responses (PR),", and "work concerns (PW)" of the stress dimensions. Moreover, it did not correlate significantly with dimensions of "physical function", "bodily pain", "physical limitation", "general health", and "mental health" of health status. This seems to imply that nurses may perceive ill health when they are overloaded with assignments or have an unfavorable job allocation. Alternatively, nurses who perceived higher job stress and worse health status may have overestimated their ability to balance job-related and personal affairs. Less experienced nurses perceived greater stress, as Table 2 indicates. In other words, nurses in the psychiatric hospitals, especially younger ones, are somewhat naïve.

\section{Discussion}

\section{Greater stress and poorer health among nurses in psychiatric hospitals}

The levels of perceived stress were 3.72 and 3.88 for HS and HV hospitals in this research. Compared to previous studies, Lee reported the mean stress level of 3.8 [5] while Tang et al. the mean stress level of 3.44 [4]. Unlike previous studies in Taiwan, which were conducted in medical centers in the western part of Taiwan, this study was conducted with samples from psychiatric hospitals in the eastern part of the country. Test results indicated that nurses in these smaller and specialized hospitals perceive similar or higher stress levels compared to nurses in the medical centers [4,5]. Regardless of the size of a hospital, administrative works and standards required by the New Teaching Hospital Accreditation of Taiwan are the same for all accredited levels of hospitals. In the meantime, hospitals in Taiwan are forced to compete with the limited reimbursements from the National Health Insurance Bureau (NHIB), decreasing the number of nursing staff. As a result, nurses need to share more administrative works and take on greater patient care obligations than before. The average score on health status in this research was 60.16 , much lower compared to those reported in previous studies. For example, a National Health Interview Survey

\begin{tabular}{|c|c|c|c|c|c|c|c|c|c|}
\hline \multirow{2}{*}{$\begin{array}{l}\text { Hospital } \\
\text { Variables }\end{array}$} & \multicolumn{2}{|c|}{ State H } & \multicolumn{2}{|c|}{ Veteran H } & \multirow{2}{*}{$\begin{array}{l}\text { Hospital } \\
\text { Variables }\end{array}$} & \multicolumn{2}{|c|}{ State H } & \multicolumn{2}{|c|}{ Veteran H } \\
\hline & $n$ & $\%$ & $n$ & $\%$ & & $n$ & $\%$ & $n$ & $\%$ \\
\hline Gender & & & & & Tenure & & & & \\
\hline$F$ & 153 & $98.71 \%$ & 176 & $98.88 \%$ & 3 yrs- & 77 & $43.26 \%$ & 62 & $34.83 \%$ \\
\hline$M$ & 2 & $1.29 \%$ & 2 & $1.12 \%$ & $4-6$ yrs & 25 & $14.04 \%$ & 47 & $26.40 \%$ \\
\hline Age & & & & & $7-10$ yrs & 27 & $15.17 \%$ & 41 & $23.03 \%$ \\
\hline 25- & 35 & $22.58 \%$ & 39 & $21.91 \%$ & $11+y r s$ & 26 & $15.60 \%$ & 28 & $15.73 \%$ \\
\hline $26-30$ & 37 & $23.87 \%$ & 41 & $23.03 \%$ & Marital & & & & \\
\hline $31-35$ & 31 & $20.00 \%$ & 31 & $17.42 \%$ & Single & 91 & $58.71 \%$ & 93 & $52.25 \%$ \\
\hline $36-40$ & 27 & $17.42 \%$ & 35 & $19.66 \%$ & Married & 57 & $36.77 \%$ & 77 & $43.26 \%$ \\
\hline $41+$ & 25 & $16.13 \%$ & 32 & $17.98 \%$ & others* & 7 & $4.52 \%$ & 8 & $4.49 \%$ \\
\hline Education & & & & & $\begin{array}{l}\text { Annual } \\
\text { income }\end{array}$ & & & & \\
\hline Vocational & 3 & $1.94 \%$ & 0 & $0.00 \%$ & $600 \mathrm{~K}-$ & 92 & $59.35 \%$ & 95 & $53.37 \%$ \\
\hline College & 53 & $34.19 \%$ & 77 & $43.26 \%$ & $610-700 \mathrm{~K}$ & 29 & $18.71 \%$ & 23 & $12.92 \%$ \\
\hline Bachelor & 79 & $50.97 \%$ & 96 & $53.93 \%$ & $710-800 \mathrm{~K}$ & 15 & $9.68 \%$ & 31 & $17.42 \%$ \\
\hline Master+ & 20 & $12.90 \%$ & 5 & $2.81 \%$ & $810 \mathrm{~K}+$ & 19 & $12.29 \%$ & 29 & $16.29 \%$ \\
\hline
\end{tabular}

Table 1: Demographic description “Divorced and widowed

\begin{tabular}{|c|c|c|c|c|c|c|c|c|}
\hline \multirow{2}{*}{ Stresses } & \multicolumn{2}{|l|}{ State } & \multicolumn{2}{|c|}{ Veteran } & \multicolumn{2}{|c|}{ Difference } & \multicolumn{2}{|l|}{ Grand } \\
\hline & Mean & S.D. & Mean & S.D. & A-B & t-test & Mean & S.D. \\
\hline Personal work(PP) & 3.83 & 2.19 & 4.16 & 2.21 & -0.33 & 0.50 & 4.01 & 2.24 \\
\hline Competence(PC) & 3.40 & 1.36 & 3.15 & 1.34 & 0.25 & 0.28 & 3.27 & 1.34 \\
\hline Personal Response(PR) & 4.29 & 1.96 & 4.43 & 1.61 & -0.14 & 0.33 & 4.36 & 1.79 \\
\hline Work concerns(PW) & 3.36 & 1.72 & 3.78 & 1.35 & -0.42 & 0.78 & 3.58 & 1.50 \\
\hline Job stress(PJ) & 3.72 & 1.81 & 3.88 & 1.63 & -0.16 & 0.47 & 3.81 & 1.72 \\
\hline 1. Physical function (PF) & 80.87 & 19.63 & 81.32 & 18.02 & -0.45 & 0.15 & 81.11 & 18.89 \\
\hline 2. Role limitations, physical (RP) & 61.56 & 11.58 & 61.39 & 12.27 & 0.17 & 0.79 & 61.47 & 12.01 \\
\hline 3. Bodily pain (BP) & 66.67 & 21.23 & 67.86 & 17.65 & -1.19 & 0.50 & 67.31 & 19.26 \\
\hline 4. General health $(\mathrm{GH})$ & 50.39 & 21.56 & 47.86 & 16.68 & 2.53 & 0.39 & 49.04 & 19.02 \\
\hline 5. Vitality (VT) & 47.89 & 18.92 & 43.33 & 15.27 & 4.56 & 0.32 & 45.45 & 16.89 \\
\hline 6. Social function (SF) & 65.24 & 22.31 & 56.55 & 18.89 & 8.69 & 0.19 & 60.59 & 20.51 \\
\hline 7. Role limitations, emotion (RE) & 67.63 & 30.25 & 57.84 & 30.56 & 9.79 & 0.39 & 62.40 & 30.61 \\
\hline 8. Mental health $(\mathrm{MH})$ & 54.56 & 17.28 & 53.35 & 15.12 & 1.21 & 0.09 & 53.91 & 16.17 \\
\hline Health status & 58.10 & 20.35 & 58.69 & 18.06 & -0.59 & 0.35 & 60.16 & 19.17 \\
\hline
\end{tabular}

Table 2: Mean, standard deviations, and differences between hospitals $N=333$ ( $H S=155 ; H V=178$ ) 
Page 4 of 6

\begin{tabular}{|c|c|c|c|c|c|c|c|c|}
\hline & \multirow[t]{2}{*}{ Variable } & \multicolumn{4}{|c|}{ Stress } & \multicolumn{3}{|c|}{ Health status } \\
\hline & & $\mathbf{N}$ & Mean & S.D. & $t / F$ & Mean & S.D. & $t / F$ \\
\hline \multirow[t]{2}{*}{ Hospitals } & $\mathrm{HS}$ & 155 & 3.84 & 1.21 & -0.68 & 55.65 & 17.69 & 1.51 \\
\hline & $\mathrm{HV}$ & 178 & 3.92 & 1.06 & & 52.69 & 14.67 & \\
\hline \multirow[t]{5}{*}{ Age } & $25-$ & 74 & 3.91 & 1.02 & 1.66 & 58.01 & 16.98 & 1.36 \\
\hline & $26-30$ & 78 & 3.98 & 1.15 & & 53.12 & 17.05 & \\
\hline & $31-35$ & 62 & 3.79 & 1.13 & & 50.28 & 17.06 & \\
\hline & $36-40$ & 62 & 3.84 & 1.12 & & 51.25 & 17.85 & \\
\hline & $41+$ & 57 & 3.18 & 1.32 & & 56.86 & 15.83 & \\
\hline \multirow[t]{4}{*}{ Education } & Vocational & 3 & 4.22 & 1.14 & 1.10 & 50.01 & 24.49 & $3.218^{*}$ \\
\hline & College & 130 & 3.87 & 0.97 & & 48.66 & 17.33 & \\
\hline & University & 175 & 3.78 & 1.17 & & 55.65 & 17.12 & \\
\hline & Master & 25 & 3.05 & 1.03 & & 65.57 & 12.04 & \\
\hline \multirow[t]{4}{*}{ Tenure } & 3- & 139 & 3.86 & 1.10 & $3.137^{*}$ & 57.15 & 16.56 & $3.286^{\star}$ \\
\hline & $4 \sim 6$ & 72 & 4.01 & 1.26 & & 51.28 & 17.43 & \\
\hline & $7 \sim 10$ & 68 & 3.96 & 0.98 & & 49.95 & 17.08 & \\
\hline & $11+$ & 54 & 3.32 & 1.09 & & 50.18 & 17.16 & \\
\hline \multirow[t]{3}{*}{ Units } & Acute & 142 & 3.88 & 0.87 & $4.745^{* \star *}$ & 52.75 & 18.04 & 1.93 \\
\hline & Chronic & 96 & 4.25 & 1.23 & & 51.04 & 15.83 & \\
\hline & LTC $^{a}$ & 95 & 3.36 & 1.53 & & 60.25 & 17.72 & \\
\hline \multirow[t]{3}{*}{ Shift } & Day & 143 & 3.62 & 1.15 & $2.979^{*}$ & 53.69 & 16.58 & 2.21 \\
\hline & Night & 64 & 4.16 & 1.23 & & 49.16 & 17.93 & \\
\hline & Rotate & 126 & 3.93 & 1.17 & & 52.27 & 17.68 & \\
\hline \multirow[t]{3}{*}{ Marital } & Single & 184 & 4.09 & 1.10 & $7.861^{* *}$ & 51.86 & 18.33 & 2.51 \\
\hline & Married & 134 & 3.46 & 1.20 & & 58.35 & 17.52 & \\
\hline & others & 15 & 3.98 & 0.56 & & 67.53 & 14.01 & \\
\hline \multirow[t]{4}{*}{ Children } & 0 & 210 & 4.08 & 1.10 & $16.023^{* *}$ & 52.01 & 17.56 & 2.74 \\
\hline & 1 & 54 & 3.69 & 1.38 & & 61.42 & 17.63 & \\
\hline & 2 & 48 & 3.63 & 1.26 & & 55.41 & 16.31 & \\
\hline & $3+$ & 21 & 4.15 & 1.40 & & 52.25 & 18.86 & \\
\hline \multirow[t]{4}{*}{ Yearly salary } & $60 \mathrm{~K}-$ & 187 & 3.94 & 1.13 & 1.29 & 54.21 & 16.68 & 0.22 \\
\hline & $61-70 \mathrm{~K}$ & 52 & 3.71 & 1.35 & & 52.26 & 21.23 & \\
\hline & $71-80 \mathrm{~K}$ & 48 & 3.84 & 1.28 & & 52.33 & 18.71 & \\
\hline & $81 \mathrm{~K}+$ & 46 & 3.49 & 0.90 & & 50.89 & 14.63 & \\
\hline
\end{tabular}

Table 3: Stress and health status by demographic characteristics a LTC, Long-term care, $p<0.05^{, ~ * *} p<0.0^{1, * * *} p<0.001$

reported average scores of 70.7 for men and 75.2 for women [22]; Lee et al. reported an average score of 73.35 in a health-related quality of life study [23]; and Wang et al. indicated a slightly lower health score of 65.85 [24]. Given that the score of 70 indicates a better health status [22], nearly 80 percent of nurses in these psychiatric hospitals are in poor health. This is definitely a risky factor for the quality of health service provided to patients and for the associated costs to the hospitals; therefore, it deserves special attention.

The current research shows that nurses are not significantly different in stress and health status in terms of demographic factors and job characteristics. This means no matter what kind of personal characteristics and job nature will be, the nurses in psychiatric hospitals indifferently perceived stronger stress and poorer health status.

\section{Avoidance approach may not work to cope with stress}

The hospitals selected for this study were situated in an excellent tourism destination. Plenty of natural resources and leisure facilities are in the neighborhood and are highly accessible in terms of geographic distance and purchasing power. Compared to those who work in the hospitals in the western metropolitans, nurses in the case hospitals are in a superior position of easy access to utilize tourism resources to cope with stress. In fact, the current research shows that nurses in the hospitals located in the eastern part of the country experience greater stress and poorer health compared to their counterparts in the west, as the previous section has discussed. Despite that the family obligation may also contribute additional effects to greater stress and poorer health, as the next paragraph will discuss, the avoidance strategy, such as escaping to the tourism activities, may not bring sustainable effects for revitalization.

\section{Family obligation is magnified in eastern Taiwan}

Consistent with a previous study [23], the current study showed that respondents who have worked in the hospital for 15 or more years perceived less stress compared to younger nurses. Family life and associated family-work conflicts could be a source of stress $[13,14,18]$. Current research provides additional evidence by showing that the married nurses with two or three children perceived greater stress compared to those who were single or had no children. This is particularly true in traditional Chinese society, where women are expected to assume most of the responsibility for the house, children, and older adults. This group of nursing professionals tends to exaggerate the most impactful forces of family obligation because of their selfperception of socio-economic status in such a regional sub-culture in Taiwan. 


\begin{tabular}{|c|c|c|c|c|c|c|c|c|c|c|c|c|c|}
\hline Dimensions & PJ & PP & PC & PR & PW & HP & 1 & 2 & 3 & 4 & 5 & 6 & 7 \\
\hline Job stress(PJ) & 1 & & & & & & & & & & & & \\
\hline Personal affair(PP) & $0.308^{* *}$ & 1 & & & & & & & & & & & \\
\hline Competence (PC) & $0.326^{* *}$ & -0.176 & 1 & & & & & & & & & & \\
\hline Personal response (PR) & $0.497^{\text {t* }}$ & $0.556^{* *}$ & 0.106 & 1 & & & & & & & & & \\
\hline Work concern (PW) & $0.385^{* *}$ & $0.547^{* *}$ & 0.006 & $0.715^{* *}$ & 1 & & & & & & & & \\
\hline Perceived health(HP) & $-0.406^{* *}$ & $-0.365^{* *}$ & $-0.287^{* *}$ & $-0.696^{* *}$ & $-0.513^{* *}$ & 1 & & & & & & & \\
\hline PF (function) & $-0.184^{* *}$ & $-0.196^{* *}$ & -0.051 & $-0.223^{* *}$ & -0.147 & $0.557^{* *}$ & 1 & & & & & & \\
\hline RP (physical) & $-0.312^{* *}$ & $-0.339^{* *}$ & -0.077 & $-0.504^{* *}$ & $-0.361^{* *}$ & $0.785^{* *}$ & $0.446^{* *}$ & 1 & & & & & \\
\hline $\mathrm{BP}$ (pain) & $-0.188^{* *}$ & $-0.426^{* *}$ & -0.028 & $-0.561^{* *}$ & $-0.274^{* *}$ & $0.642^{* *}$ & $0.454^{*}$ & $0.366^{* *}$ & 1 & & & & \\
\hline GH (general) & $-0.282^{* *}$ & $-0.345^{* *}$ & -0.056 & $-0.485^{* *}$ & $-0.291^{* *}$ & $0.622^{* *}$ & $0.265^{* *}$ & $0.384^{* *}$ & $0.470^{* *}$ & 1 & & & \\
\hline VT (vital) & $-0.358^{* *}$ & $-0.377^{\star *}$ & $-0.285^{\star *}$ & $-0.679^{* *}$ & $-0.338^{* *}$ & $0.732^{* *}$ & $0.257^{\star \star}$ & $0.455^{* *}$ & $0.472^{+*}$ & $0.451^{* *}$ & 1 & & \\
\hline SF (social) & $-0.228^{* *}$ & $-0.413^{* *}$ & $-0.295^{\star *}$ & $-0.468^{* *}$ & $-0.376^{* *}$ & $0.774^{* *}$ & $0.348^{* *}$ & $0.510^{* *}$ & $0.583^{* *}$ & $0.439^{* *}$ & $0.588^{* *}$ & 1 & \\
\hline RE (emotion) & $-0.336^{* *}$ & $-0.205^{* *}$ & $-0.287^{* *}$ & $-0.511^{* *}$ & $-0.398^{* *}$ & $0.783^{* *}$ & $0.267^{* \star}$ & $0.589^{* *}$ & $0.236^{* *}$ & $0.327^{* *}$ & $0.474^{* *}$ & $0.428^{* *}$ & 1 \\
\hline MH (mental) & $-0.312^{* *}$ & -0.151 & -0.126 & $-0.415^{\star *}$ & $-0.308^{* *}$ & $0.332^{* *}$ & 0.163 & $0.286^{* *}$ & 0.158 & 0.147 & $0.254^{* *}$ & $0.263^{* *}$ & $0.295^{* *}$ \\
\hline
\end{tabular}

Table 4: Correlation of stress and health status, $N=333 ;{ }^{*} p<0.05,{ }^{* *} p<0.01,{ }^{* * *} p<0.001$

Despite the fact that nurses in the eastern part of the country perceive greater stress compared to nurses in the western part [4,5], turnover rates in this area are lower. Noteworthy is that nurses in both parts of the country are trained by institutes under same education system, and are employed by the facilities under the same accreditation system. This suggests the presence of regional subculture. Part of the reason behind this phenomenon is due to the restricted availability of job offers in the east [17]. Another reason may stem from the perception of the socioeconomic status of nursing professionals in Eastern Taiwan's society as well as the nurses' perceived psychological contract [25] with the hospitals. The medical professionals in the Eastern Taiwan generally receive a better compensation package (similar levels of compensation plus a special allowance). Job positions with responsibility for human life offer attractive incomes, which have given the medical profession a highly respected socio-economic status in the less-developed east. As a result, nurses may perceive even stronger stress from their family obligations, for example, a better educational performance of their children, or a nicer housekeeping among others. This means that the levels of the psychological contract that bind the nurses' subjective perception of their family obligations may be stronger for those in the eastern rather than western part of the country. This may imply that nurses in Eastern Taiwan may perceive stronger family obligations because they are viewed as having higher socioeconomic status.

Since a supportive culture increases job satisfaction and organizational commitment [26], the hospitals should start establishing and maintaining a supportive atmosphere with a focus on balancing work and family obligations to enhance person-organization fit $[13,14,26]$. Special care is needed in a particular subculture.

\section{Conclusion}

The nurses in the hospitals that were included in this study did not perceive stress and health status significantly differently. These hospitals fare worse compared to those in the western part of Taiwan in terms of turnover, costs, and service quality. Thus, the nursing professionals in the eastern psychiatric hospitals are expected to be exposed to higher than average stresses, increasing their risks of both poor person-environment fit and organizational commitment. The "personal response" has the greatest association with almost every aspect of poor health status and thus needs special attention. Despite the compensation package and the social status associated with being a nursing professional, the respondents perceived not only the stresses from their jobs, but also stresses from family and society, and consequently, they experienced poorer health status. Unless the nurses engage in stress-coping activities, stresses may not subside. Nurses have manifold opportunities to engage in stress-relieving activities because the hospital is situated in a district surrounded by abundant leisure or sightseeing resources. Eastern Taiwan is well known for its excellent natural resources and tourism sites. However, precious resources of this kind may not be relied on as sources of stress relief. Administrators at all levels of the hospitals should establish and maintain an effective and easily accessible support system to enhance the nurses' organizational commitment and properly address the individual-specific stress sources (i.e., family and society in particular). Eventually, this may foster a person-environment fit. The higher the degree of person-environment fit, the higher the job satisfaction and the better the health status.

Excess stress may be detrimental to health. Although the current research showed a moderate level of relationship between perceived stress and health status, personal response to stress has a detrimental effect on health. The professionals are well compensated with generous allowances. As a result, these female professionals are forced by the traditional culture to meet high standard in keeping or managing her family. This implies that effective stress management strategies should be customized to specific needs of each individual. A periodical and close monitoring of each individual nurse's family obligation would be able to generate effective and timely advices or assistance.

This research is among the first to link the concept of personenvironment fit with nurses' stress and one of the first to consider social factors in the PE fit. Thus, it may contribute to our understanding of stress and health among nurses, particularly those working in psychiatric hospitals. First, a generous compensation program may merely satisfices the psychiatric nurses' basic need. Second, the current research provides evidence for the deficiency of an avoidance strategy. This implies that hospitals cannot rely solely on locating a tourism destination to help workers' deal with stress. Third, social and economic status in a society can impose additional pressure on the workers. Fourth, the social desirability of the captioned environment can affect the levels of person-environment.

\section{Limitations and Future Research Recommendation}

Although the sample distribution in this research was not significantly different from the national data, the results may not be fully representative of the population and thus need to be interpreted 
Citation: Pan FC, Chen SJ (2015) Exploring the Stresses and Health of the Nurses in the Psychiatric Hospitals in Eastern Taiwan: Does the Leisure Destination Location Help Nurses Cope with Stress?. J Psychiatry 18: 277 doi:10.4172/psychiatry. 1000277

Page 6 of 6

carefully. Other limitations are that the study did not establish the causal relationship between perceived stress and health status. Furthermore, the effects of social desirability on the levels of PE fit were not statistically different, and they need to be empirically tested in the future. Additionally, health status was measured by subjective grading using a self-administered questionnaire. Accordingly, additional objective measures may be needed to support the real health status of the respondents.

\section{References}

1. Waldman JD, Kelly F, Aurora S, Smith HL (2004) The shocking cost of turnover in health care. Health Care Manage Rev 29: 2-7.

2. Rothmann S, Van der Colff JJ, Rothmann JC (2014) Occupational stress of professional nurses in South Africa. Curationis 24: 375-384.

3. Kawano Y (2008) Association of job-related stress factors with psychological and somatic symptoms among Japanese hospital nurses: effect of departmental environment in acute care hospitals. J Occup Health 50: 79-85.

4. Tang PL, Chen WL, Chen HF (2005) Depression level and its associated factors in nurses. Formosa J Ment Health 18: 55-74.

5. Lee I (2004) Work stress, coping strategies, and consequences among public health nurses - based on an interactive model. Taiwan Journal Public Health 23: $398-405$

6. Lambert VA, Lambert CE, Petrini M, Li XM, Zhang YJ (2007) Predictors of physical and mental health in hospital nurses within the People's Republic of China. Internat Nurs Rev 54: 85-91.

7. Gandhi S, Sangeetha G, Ahmed N, Chaturvedi SK (2014) Somatic symptoms, perceived stress and perceived job satisfaction among nurses working in an Indian psychiatric hospital. Asian journal of psychiatry 12: 77-81.

8. Van der Heijden BIJM, Demerouti E, Bakker AB (2008) Work-home interference among nurses: Reciprocal relationships with job demands and health. J Adv Nurs 62: 572-584.

9. Day G, Minichiello V, Madison J (2010) Nursing morale: predictive variables among a sample of registered nurses in Australia. J Nurs Manag 15: 274-284.

10. Deklava L, Circenis K, Millere I (2014) Stress coping mechanisms and professional burnout among Latvian nurses. Procedia-Social and Behav Sc 159: 261-267.

11. Hayes LJ, O’Brien-Pallas L, Duffield C (2012) Nurse turnover: A literature review-An update. Int J Nursing Studies 49: 887-905.
12. Lazarus RS (2000) Toward better research on stress and coping. Am Psychol 55: 665- 673 .

13. Chuang AC, Hsu SW, Wang A, Judge TA (2014) Does west "fit" with east? In search of a Chinese model of person-environment fit. Academy of Management Journal, in press.

14. Schneider B (1987) the people make the place. Personnel Psychology 40: 437-453.

15. Muchinsky PM, Monahan CJ (1987) What is person-environment congruence? Supplementary versus complementary models of fit. J Vocational Behav 31 268-277.

16. Schneider B (2001) Fits about fit. Appl Psychol 50: 141-152.

17. Taiwan Union of Nurse Association (2014)

18. Benoliel JQ, McCorkle R, Georgiadou F, Denton T, Spitzer A (1990) Measurement of stress in clinical nursing. Cancer Nursing 13: 221-228.

19. Tsai SL, Chen ML (1996) A test of the reliability and validity of Nurse Stress Checklist. The Journal of Nursing Research 4: 355-361.

20. Lu JFR, Tseng HM, Tsai YJ (2003) Assessment of health-related quality of life in Taiwan (I): development and psychometric testing of SF-36 Taiwan version. Taiwan Journal Public Health 22: 501-511.

21. Tseng HM, Lu JFR, Tsai YJ (2003) Assessment of health-related quality of life in Taiwan (I I): Norming and validation of SF-36 Taiwan version. Taiwan Journal Public Health 22: 512-518.

22. Chung W-SI, Chang HY, Shih Y-T, Wen C-P (2003) Self-perceived mental health of Taiwan residents: Result of 2001 national health interview survey Taiwan Journal Public Health 22: 465-473.

23. Lee PH, Chang W, Tsai JC (2005) A study of physical fitness and health-related quality of life among nursing staff. New Taipei Journal Nursing 7: 53-63.

24. Wang $\mathrm{CH}$, Lee PH, Jeng C (2006) A study of fatigue/stamina, healthy lifestyle and health-related quality of life among nurses. New Taipei Journal Nursing 8 : 7-16.

25. Rousseau DM (1989) Psychological and implied contracts in organizations Employee Responsibilities and Rights Journal 2: 121-139.

26. Silverthorne C (2004) The impact of organizational culture and personorganization fit on organizational commitment and job satisfaction in Taiwan. Leadership \& Organization Development Journal 25: 592-599. 